\title{
A quantitative link between microplastic instability and macroscopic deformation behaviors in metallic glasses
}

\author{
Y. Wu, ${ }^{1}$ G. L. Chen, ${ }^{1}$ X. D. Hui, ${ }^{1}$ C. T. Liu, ${ }^{2}$ Y. Lin, ${ }^{2}$ X. C. Shang, ${ }^{3}$ and Z. P. Lu ${ }^{1, a)}$ \\ ${ }^{1}$ State Key Laboratory for Advanced Metals and Materials, University of Science and Technology Beijing, \\ Beijing 100083, People's Republic of China \\ ${ }^{2}$ Department of Mechanical Engineering, Hong Kong Polytechnic University, Hung Hom, Kowloon, \\ Hong Kong \\ ${ }^{3}$ Department of Mathematics and Mechanics, University of Science and Technology Beijing, Beijing 100083, \\ People's Republic of China
}

(Received 14 July 2009; accepted 18 September 2009; published online 22 October 2009)

\begin{abstract}
Based on mechanical instability of individual shear transformation zones (STZs), a quantitative link between the microplastic instability and macroscopic deformation behavior of metallic glasses was proposed. Our analysis confirms that macroscopic metallic glasses comprise a statistical distribution of STZ embryos with distributed values of activation energy, and the microplastic instability of all the individual STZs dictates the macroscopic deformation behavior of amorphous solids. The statistical model presented in this paper can successfully reproduce the macroscopic stress-strain curves determined experimentally and readily be used to predict strain-rate effects on the macroscopic responses with the availability of the material parameters at a certain strain rate, which offer new insights into understanding the actual deformation mechanism in amorphous solids.
\end{abstract}

(C) 2009 American Institute of Physics. [doi:10.1063/1.3247968]

\section{INTRODUCTION}

Understanding deformation mechanism of metallic glasses has been advanced significantly but still remained to be an attracting challenge for the field at the present time. ${ }^{1-7}$ The theoretical foundation for explaining plastic deformation behavior of metallic glasses is the classical concept of shear transformation zones (STZs). ${ }^{2-4,6,7}$ Based on this conception, molecular dynamic simulations have been conducted to describe the deformation behavior of two-dimensional ${ }^{4,8}$ or three-dimensional glasses. ${ }^{9}$ Restricted by the calculation capability, such simulations were limited to rather simple, small systems. Recently, Johnson and Samwer ${ }^{3}$ extended the STZ scheme by combining it with the concept of potential energy landscapes, and shearing of a glassy solid is basically regarded as a sum of highly localized shear events, which are triggered when the elastic energy inside individual STZ reaches their corresponding energy barriers for plastic shearing. Macroscopic yielding occurs when a critical fraction of STZs are activated. Based on the average mechanical instability of STZs, Johnson and Samwer ${ }^{3}$ successfully established a universal relationship between plastic yielding and glass transition temperature of metallic glasses. However, to the best of our knowledge, no available model can give a complete description of the deformation behavior at different length scales. As pointed out clearly by Barrat and de Pablo, ${ }^{10}$ a more realistic model capable of bridging the gap between localized plasticity of STZs and global deformation behavior is urgently needed.

More recently, both compositional and structural heterogeneities such as short range and medium-range orderings,

\footnotetext{
${ }^{a)}$ Author to whom correspondence should be addressed. Electronic mail: luzp@skl.ustb.edu.cn.
}

clusters, etc., in metallic glasses have been confirmed and widely studied. ${ }^{11-15}$ Increasing simulation and experimental results suggested that these heterogeneities respond differently under stress and the relevant microinstabilities of local rearrangements may ${ }^{16-18}$ play important roles in the macroscopic mechanical properties of amorphous solids. All these observations suggest that STZs in actual macroscopic amorphous materials have a statistical distribution of activation energy barriers corresponding to different local atomic packing and orientation, as already noted by Johnson and Samwer. ${ }^{3}$ In this paper, therefore, we attempt to build up a quantitative link between microplastic instability of STZs with distributed activation energy barriers and the macroscopic deformation behavior (e.g., the stress-strain relationship) for metallic glasses.

\section{EXPERIMENTAL}

To demonstrate and validate our current model, glassy specimens at multiple length scales including the $\mathrm{Co}_{69.5} \mathrm{Fe}_{4.5} \mathrm{Cr}_{1} \mathrm{Si}_{8} \mathrm{~B}_{17}$ amorphous wires with a diameter ranging from 30 to $190 \mu \mathrm{m}$ and a bulk $\mathrm{Zr}_{53.7} \mathrm{Cu}_{28.5} \mathrm{Ni}_{9.4} \mathrm{Al}_{8.4}$ glassy sheet with a thickness of $2 \mathrm{~mm}$ were prepared. The details regarding the sample preparation and tensile testing of the small sized Co-based specimens can be found elsewhere. ${ }^{19}$ Dog bone-shaped samples of the Zr-based glass with a gauge dimension of $10 \times 0.48 \times 1.0 \mathrm{~mm}^{3}$ were cut from a plate fabricated by suction-casting high-purity constituents. Tensile tests of the Zr-based glassy specimens were carried out in a Reger 3000A testing machine at a strain rate of $2 \times 10^{-4} \mathrm{~s}^{-1}$. Nanoindentation experiments were conducted using a MTS dynamic control module (DCM) nanoindentation system at various loading rates ranged from $8 \times 10^{-3}$ to $2 \times 10^{-1} \mathrm{~s}^{-1}$. The numerical calculations were performed in the MATLAB program. 


\section{RESULTS}

\section{A. Model construction}

In our approach, metallic glasses were regarded as an ensemble of numerous STZ embryos which can be possibly activated to take plastic shear events with a finite activation energy. Due to the lack of periodic structure, the random energy model is adopted which describes the energy levels in a disordered system as independent random variables. The populations of STZs with different activation energy $v$ for the occurrence of plastic instability are assumed to follow the quasi-Gaussian distribution $^{20}$

$$
p(v)=\left\{\begin{array}{ll}
p_{0} \exp \left[-\frac{(v-\bar{V})}{2 \Sigma^{2}}\right] & (v \geq 0) \\
0 & (v<0)
\end{array}\right\},
$$

where $\bar{V}$ is the apparent average activation energy of the sample, $\Sigma$ is the standard deviation of activation energies, and $p_{0}$ is a prefactor. Obviously, the probability density function $p(v)$ satisfies the normalization condition: $\int_{0}^{\infty} p(v) d v=1$. Activation of the STZs was taken as a stress-assisted thermal activation process and the activation rate reads $\Gamma(v)$ $=\gamma \exp \left(-v / k_{B} T\right)$, where $\gamma$ is a prefactor independent of energy and temperature $T$, and $k_{B}$ is the Boltzmann constant.

As indicated by previous simulation results, ${ }^{15}$ both thermal and mechanical energy contribute to the activation process of the STZs. Along with the external applied stress, STZs with smaller activation energies can be more easily activated to take some local atomic rearrangement and produce a certain plastic strain. After the activation, all the atoms inside the entire amorphous solid turn into a new configuration state (new STZs could be formed due to the atomic redistribution) and remeshed into different STZs ready for the next activation event.

As the deformation proceeds (i.e., the applied stress is increased), STZs with large activation energies are progressively activated, resulting in an appreciable plastic strain in the macroscopic stress-strain curve. Mathematically, the strain tensor of any individual STZ was divided into the tensors for elastic and plastic parts, i.e., $\boldsymbol{\varepsilon}(t)=\boldsymbol{\varepsilon}_{e}(t)+\boldsymbol{\varepsilon}_{p}(t)$. Assume that once the STZ is activated to take a plastic instability at a random time $\tau \in[0, t]$, all the deformation in the STZ turns plastic and becomes irreversible, that is, $\left.\boldsymbol{\varepsilon}_{p}(t, \tau)\right|_{t=\tau}=\boldsymbol{\varepsilon}(\tau)$. In addition, deformation of STZs can be taken as viscoplastic ${ }^{4}$ because of their sufficiently small volume, and the rate-of-strain tensor for plastic deformation is proportional to the rate-of-strain tensor for the total deformation, namely $d \varepsilon_{p}(t) / d t=\phi(t)[d \varepsilon(t) / d t]$. The function $\phi(t)$, $\phi(t)=1-\exp \left\{-\alpha\left[\varepsilon(t)-\varepsilon_{p}(t)\right]^{\beta}\right\}$, is similar to the so-called Matsuoka equation ${ }^{21}$ often used in amorphous polymers, where $\alpha$ is a parameter relating to the elastic strain at the apparent yielding point, $\beta$ serves as a measure of nonlinearity of the mechanical behavior and is analogous to the stretch exponent of viscous relaxation at the glass transition temperature.
As mentioned earlier, activation of STZs is a dynamic rearrangement process which can be described by the function $n(t, \tau, v)$ that is the number of STZs at time $t$ with activation energy $v$, which have last rearranged before instant $\tau \in[0, t]$. In particular, $n(t, t, v)$ is the number of STZs with activation energy $v$ at time $t,\left.n(t, \tau, v)\right|_{\tau=t}=n_{0}(v)=N p(v)$, where $N$ is denoted as the total number of STZs in the sample. After some algebraic operations, one can get

$$
\begin{aligned}
& n(t, 0, v)=N p(v) \exp [-\Gamma(v) t] \\
& \frac{\partial n}{\partial \tau}(t, \tau, v)=N p(v) \Gamma(v) \exp [-\Gamma(v)(t-\tau)] .
\end{aligned}
$$

Unlike noncrystalline polymers that can be taken as an incompressible material, numerous experiments have shown that dilatation and nanovoids could be created during tensile deformation of metallic glasses. ${ }^{19,22,23}$ As such, both shear and volumetric strain components should be considered when calculating the strain energy density ${ }^{24}$ stored in each STZ

$$
W(t)=\frac{1}{2} \lambda\left\{\operatorname{tr}\left[\boldsymbol{\varepsilon}_{e}(t)\right]\right\}^{2}+\mu \boldsymbol{\varepsilon}_{e}(t): \boldsymbol{\varepsilon}_{e}(t),
$$

where $\lambda$ and $\mu$ are Lame coefficients, denotes convolution of tensors. In the above equation, the item $\frac{1}{2} \lambda\left\{\operatorname{tr}\left[\boldsymbol{\varepsilon}_{e}(t)\right]\right\}^{2}$ represents the volumetric strain energy the density which usually equals to zero in an incompressible solid, and $\mu \boldsymbol{\varepsilon}_{e}(t): \boldsymbol{\varepsilon}_{e}(t)$ is shear strain energy density. From the strain energy density per STZ and the number of STZs with activation energy $v$ in the sample, the average strain energy density of the sample can be obtained as

$$
\begin{aligned}
W_{\mathrm{ave}}(t)= & \frac{1}{N} \int_{0}^{\infty}\left\{n(t, 0, v)\left[\frac{1}{2} \lambda\left\{\operatorname{tr}\left[e_{e}^{0}(t)\right]\right\}^{2}+\mu e_{e}^{0}(t): e_{e}^{0}(t)\right]\right. \\
& +\int_{0}^{t} \frac{\partial n}{\partial \tau}(t, \tau, v)\left[\frac{1}{2} \lambda\left\{\operatorname{tr}\left[e_{e}(t, \tau)\right]\right\}^{2}\right. \\
& \left.\left.+\mu e_{e}(t, \tau): e_{e}(t, \tau)\right] d \tau\right\} d v
\end{aligned}
$$

where $e_{e}^{0}(t)$ and $e_{e}(t, \tau)$ are the elastic strain tensors of the STZs that remain unactivated and have been activated at time $\tau \in[0, t]$, respectively. To make a connection between the stress and strain, recall that the Clausius-Duhem inequality takes the form

$$
Q(t)=-\frac{d W_{\mathrm{ave}}}{d t}(t)+\boldsymbol{\sigma}(t): \frac{d \boldsymbol{\varepsilon}}{d t}(t) \geq 0,
$$

where $Q(t)$ stands for energy dissipation of the average STZ per unit time and unit volume, $\boldsymbol{\sigma}(t)$ is the stress tensor at time $t$. Notice that Eq. (5) must hold for arbitrary strain state which, in light of Eqs. (2) and (4), leads to 


$$
\begin{aligned}
& \frac{1}{3} \operatorname{tr}[\boldsymbol{\sigma}(t)]= \lambda[1-\phi(t)]\left\{\operatorname{tr}\left[\boldsymbol{\varepsilon}(t)-\boldsymbol{\varepsilon}_{p}(t)\right]-\int_{0}^{\infty} p(v) \Gamma(v) d v\right. \\
&\left.\times \int_{0}^{t} \exp [-\Gamma(v)(t-\tau)] \operatorname{tr}\left[\boldsymbol{\varepsilon}(\tau)-\boldsymbol{\varepsilon}_{p}(\tau)\right] d \tau\right\} \\
& \boldsymbol{\sigma}^{\prime}(t)=2 \mu[1-\phi(t)]\left\{\left[\boldsymbol{\varepsilon}(t)-\boldsymbol{\varepsilon}_{p}(t)\right]-\int_{0}^{\infty} p(v) \Gamma(v) d v\right. \\
&\left.\times \int_{0}^{t} \exp [-\Gamma(v)(t-\tau)]\left[\boldsymbol{\varepsilon}(\tau)-\boldsymbol{\varepsilon}_{p}(\tau)\right] d \tau\right\}
\end{aligned}
$$

in which $\boldsymbol{\sigma}^{\prime}$ is the deviatoric part of the stress tensor. Under uniaxial tension, the total and plastic strain tensors take the simple forms

$$
\begin{aligned}
& \boldsymbol{\varepsilon}(t)=\varepsilon(t)\left[e_{\mathbf{1}} \otimes e_{\mathbf{1}}-\nu\left(e_{\mathbf{2}} \otimes e_{\mathbf{2}}+e_{\mathbf{3}} \otimes e_{3}\right)\right], \\
& \boldsymbol{\varepsilon}_{p}=\varepsilon_{p}(t)\left[e_{\mathbf{1}} \otimes e_{\mathbf{1}}-\nu_{p}\left(e_{\mathbf{2}} \otimes e_{\mathbf{2}}+e_{\mathbf{3}} \otimes e_{\mathbf{3}}\right)\right],
\end{aligned}
$$

where $e_{k}(k=1,2,3)$ are unit vectors of a Cartesian coordinate frame, whose basis vector $e_{1}$ coincides with the direction of deformation, and $\otimes$ denotes the tensor product. In the case of small plastic deformation such as in metallic glasses, Poisson's ratio for the plastic deformation $\nu_{p}$ is approximately equal to the Poisson's ratio for the bulk material $\nu$. Thus, the stress-strain relationship can be obtained as

$$
\begin{aligned}
\sigma(t)= & \frac{1}{3} \operatorname{tr}[\boldsymbol{\sigma}(t)] \mathbf{I}+\boldsymbol{\sigma}^{\prime}(t) \\
= & E[1-\phi(t)]\left\{\left[\varepsilon(t)-\varepsilon_{p}(t)\right]-\int_{0}^{\infty} p(v) \Gamma(v) d v\right. \\
& \left.\times \int_{0}^{t} \exp [-\Gamma(v)(t-\tau)]\left[\varepsilon(\tau)-\varepsilon_{p}(\tau)\right] d \tau\right\},
\end{aligned}
$$

where $E$ is the elastic modulus. As such, Eq. (8) actually has linked the localized atomic packing characteristics (corresponds to different microplastic instability) with the macrodeformation behavior of metallic glasses.

\section{B. Activation energy of STZs}

In order to describe the macroscopic deformation behavior, several parameters in Eq. (8), particularly the distribution of activation energy of STZs, have to be determined in advance. In a typical stress relaxation experiment, the evolution of macroscopic stress $\sigma(t)$, according to Eq. (8), is given by

$$
\bar{\sigma}(t)=1-\int_{0}^{\infty} p(v)[1-\exp (-\Gamma(v) t)] d v
$$

where $\bar{\sigma}(t)=\sigma(t) / \sigma^{0}$ and $\sigma^{0}$ is the stress at the beginning of the relaxation. Hence important parameters in our model can be estimated by fitting the experimental data with Eq. (9). Substituting these parameters into Eq. (1), distribution of the activation energy of the STZs in the sample can then be estimated.

Stress relaxation experiments were conducted at a preset strain of $2.5 \%$ for the $\mathrm{Co}_{69.5} \mathrm{Fe}_{4.5} \mathrm{Cr}_{1} \mathrm{Si}_{8} \mathrm{~B}_{17}$ amorphous wires
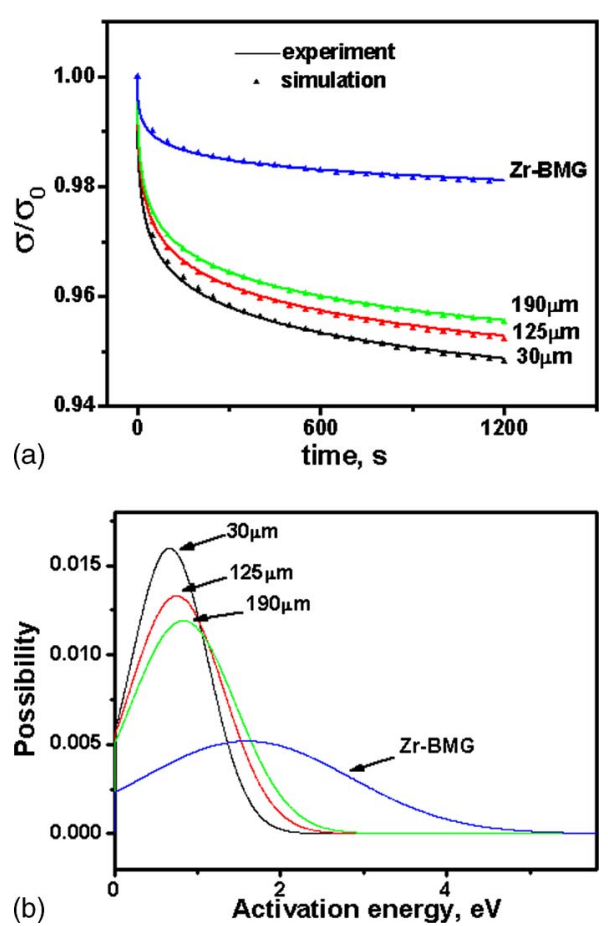

FIG. 1. (Color online) Stress relaxation responses (solid line) and fitting results (circles) with Eq. (9) of the $\mathrm{Co}_{69.5} \mathrm{Fe}_{4.5} \mathrm{Cr}_{1} \mathrm{Si}_{8} \mathrm{~B}_{17}$ amorphous wires with various diameters at a preset strain of $2.5 \%$ and the bulk $\mathrm{Zr}$-based glassy specimen with dimension of $10 \times 0.48 \times 1.0 \mathrm{~mm}^{3}$ at a preset strain of $1.9 \%$ (a), and the derived quasi-Gaussian distribution of the activation energy in these specimens (b).

with various diameters and at a strain of $1.9 \%$ for a $\mathrm{Zr}$-based bulk metallic glass (BMG) sample, respectively (the preset values were chosen just ahead the fracture). The corresponding experimental data and their fitting with Eq. (9) are shown in Fig. 1(a) by solid lines and symbols, respectively. The resultant distribution of the activation energy for the STZs in all these samples is shown in Fig. 1(b). Clearly, larger specimens reach the equilibrium state much quicker during the relaxation tests and also show a higher and wider distribution of the activation energy than the smaller ones. In other words, thinner specimens contain much higher proportion of STZs that can be easily reshuffled for plastic deformation. Parameters obtained for individual specimen are illustrated in Table I. It is important to point out that variation in the preset strain will bring little changes to the absolute stress relaxation data but the general trend shown in Fig. 1(b) remains unchanged (will be discussed in detail later).

To validate the activation energy concept discussed above, a recently developed experimental method ${ }^{25,26}$ was adopted to characterize the energy barrier of the STZs in the

TABLE I. Material parameters for different specimens derived from stress relaxation experiments with Eq. (9).

\begin{tabular}{lcccccc}
\hline \hline Specimen & & $\bar{V}$ & & & & \\
& & $(\mathrm{eV})$ & $\Sigma$ & $\gamma$ & $\alpha$ & $\beta$ \\
\hline $\mathrm{Co}_{69.5} \mathrm{Fe}_{4.5} \mathrm{Cr}_{1} \mathrm{Si}_{8} \mathrm{~B}_{17}$ & $30 \mu \mathrm{m}$ & 0.66 & 0.46 & 1.0 & 4.0 & 0.92 \\
& $125 \mu \mathrm{m}$ & 0.75 & 0.56 & 1.0 & 3.7 & 0.97 \\
& $190 \mu \mathrm{m}$ & 0.83 & 0.63 & 1.0 & 3.3 & 0.99 \\
$\mathrm{Zr}_{53.7} \mathrm{Cu}_{28.5} \mathrm{Ni}_{9.4} \mathrm{Al}_{8.4}-\mathbf{B M G}$ & 1.86 & 1.32 & 1.0 & 2.4 & 1 \\
\hline \hline
\end{tabular}



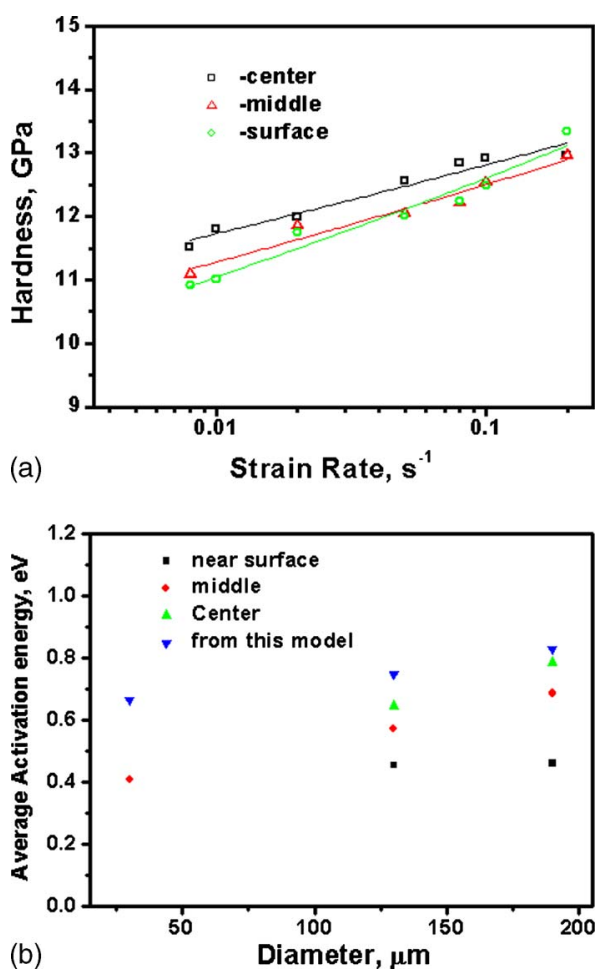

FIG. 2. (Color online) Dependence of microhardness on the strain rate at different cross-section positions of the $\mathrm{Co}_{69.5} \mathrm{Fe}_{4.5} \mathrm{Cr}_{1} \mathrm{Si}_{8} \mathrm{~B}_{17}$ amorphous wires with a diameter of $125 \mu \mathrm{m}$ (a) and the derived energy barriers in comparison with those obtained from the present model (b).

current metallic glasses. In this method, a critical parameter, namely the strain-rate sensitivity $m$, was determined from the relationship between strain rate and microhardness measured by means of nanoindentations. As an example, Fig. 2(a) shows the hardness value versus strain rate of the wire with a diameter of $125 \mu \mathrm{m}$. The strain-rate sensitivity $m$ corresponding to different cross-sectional positions of the wire (i.e., near the lateral surface, between the surface and the center, and at the center) was then determined by the slope of the linear regression line. Figure 2(b) summarizes the energy barriers of STZs measured at different positions for the 30, 125 , and $190 \mu \mathrm{m}$ wires in comparison with the average activation energy determined by our model (nanoindentation was conducted only on the position between the surface and the center in the $30 \mu \mathrm{m}$ wire because of cross-sectional area limit). According to the nanoindentation results, the measured energy barrier of STZs for the same wire is noticeably dependent on the testing position and increases from the surface to the interior, confirming that the STZ energy barrier in amorphous solids has a characteristic distribution. It is to be noticed that the average activation energy obtained by our model is in the same order but somewhat larger than that determined by molecular dynamic simulation based on the theory of potential energy landscape ${ }^{27}$ and the nanoindentation method based on the cooperative shear model. This discrepancy may be due mainly to the fact that microplastic event sizes may scale with the operational volume $e^{17,18,28}$ involved in these methods. Molecular simulation is usually limited to rather simple, one or two-component atomic sys-
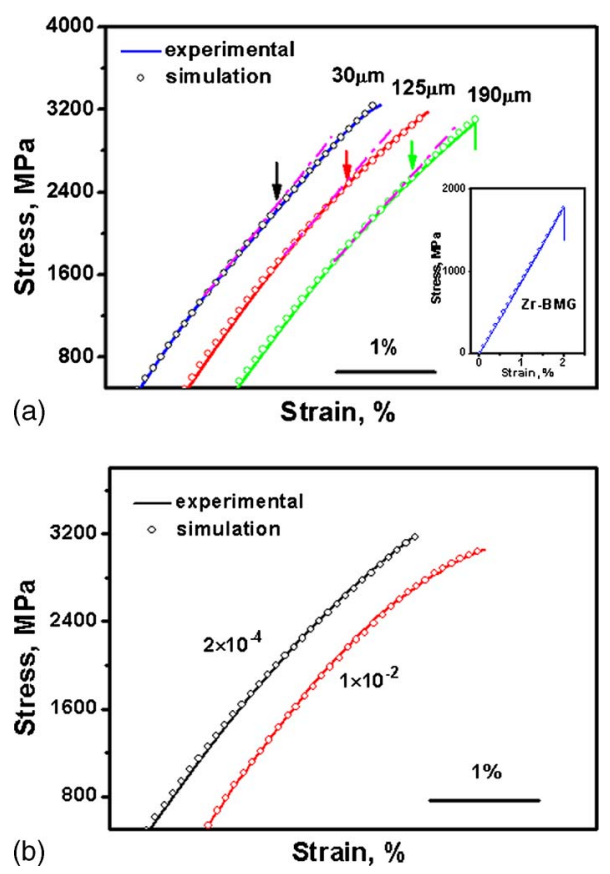

FIG. 3. (Color online) (a) Stress-strain curves of the $\mathrm{Co}_{69.5} \mathrm{Fe}_{4.5} \mathrm{Cr}_{1} \mathrm{Si}_{8} \mathrm{~B}_{17}$ amorphous wires and the bulk Zr-based glassy sample (inset), and (b) the comparison between the simulated and experimental stress-strain curves at different strain rates of the $125 \mu \mathrm{m}$ amorphous wire. Solid line indicates experimental data and empty symbols represent the fitted results with our current model.

tems, and typical indent size is no more than several microns which is far smaller than that in our stress relaxation measurements.

\section{Applications of the current model \\ 1. Modeling of the stress-strain curves}

Based on the distribution of activation energy, Eq. (8) was then used to relate the mechanical instability of STZs to the macroscopic deformation behavior in the amorphous solids. For demonstration, we have carried out tensile testing of the aforementioned Zr-based BMG bulk sample and the three micrometer sized Co-based glassy wires. Figure 3(a) shows the resultant stress-strain curves for all the samples investigated (solid lines) and the corresponding fitted ones using Eq. (8) with the parameters in Table I (empty symbols), respectively. As usual, the Zr-based bulk sample [inset in Fig. 3(a)] exhibits a linear elastic deformation behavior and a typical catastrophic fracture characteristic. However, the Cobased wire samples show an unusual nonlinear deformation with an apparent yielding phenomenon and appreciable plasticity (details about tensile properties of these wire specimens were reported elsewhere ${ }^{19}$ ). As shown in Fig. 3(a), our model can fit the experimental data not only for the nearly elastic behavior of the Zr-based bulk sample but also for the inelastic deformations of the smaller size specimens.

\section{Prediction of strain-rate effect on macroscopic deformation behavior}

Using the parameters obtained from fitting the tensile stress-strain curve at one strain rate for a certain sized sample, together with the distribution of activation energy 

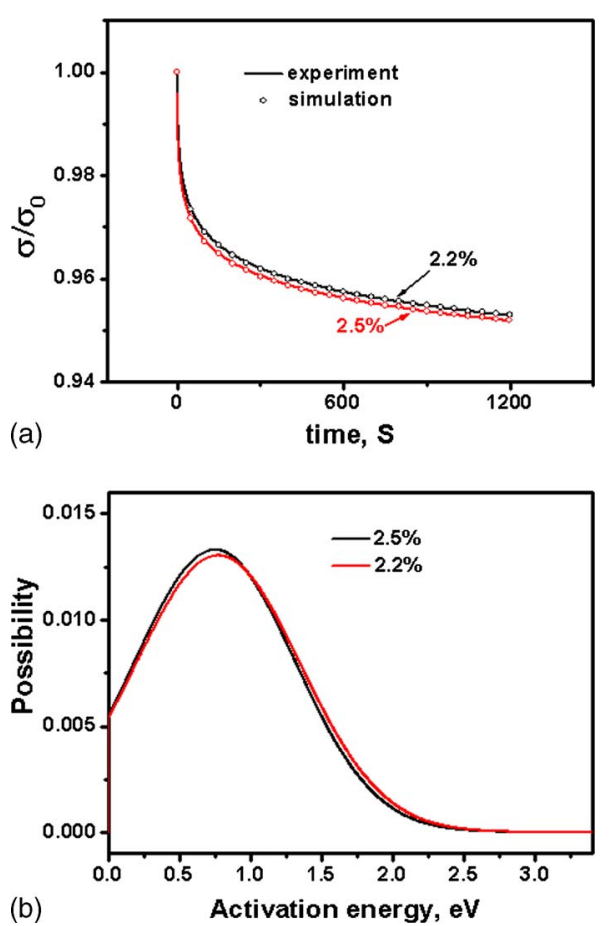

FIG. 4. (Color online) Stress relaxation responses (solid line) and fitting results (circles) of the $125 \mu \mathrm{m}$ amorphous wires under different preset strains (a), and the resultant activation energy distribution (b).

derived from the stress-relaxation experiment, tensile deformation behavior at any other strain rates can be predicted by Eq. (8). For example, with the information derived at a strain rate of $2 \times 10^{-4}$, the stress-strain curve at the strain rate of $1 \times 10^{-2}$ for the $125 \mu \mathrm{m}$ glassy wire is predicted as shown in Fig. 3(b) (circle symbol). The experimental confirmation under the same strain rate is also illustrated in Fig. 4(b) (solid line) and good agreement between the prediction and experimental data can be seen, which further validates the present model.

Similar characteristics of the deformation behaviors at different strain rates are observed in Fig. 3(b), suggesting that the rate dependence of flow stress is minor or inappreciable in plastic flow kinetics in metallic glass. ${ }^{1,2}$ However, the nonlinearity is more pronounced under the condition with a larger strain rate of $1 \times 10^{-2} \mathrm{~s}^{-1}$. It has been confirmed that the nonlinear tensile deformation of the amorphous wires may due to the coalescence of free volume which engendering nanometer voids. ${ }^{19}$ Free volume content is always determined by two competing processes: creation by the stressdriven process and the annihilation by the diffusion process. ${ }^{1}$ In the condition of higher strain rates, less time left for the diffusion and therefore more free volume available for the coalescence to form subnanometer voids, which give rises to the more pronounced nonlinear behavior at higher strain rates.

\section{DISCUSSION}

\section{A. Influencing factors of the activation energy}

As discussed above, the variation in activation energy of STZs in metallic glasses is assumed to follow a quasiGaussian distribution, from which a link between the micro- instability and macrodeformation properties is established. Similar to the Johnson-Sawmer's cooperative shear model, the distribution of the energy barrier of STZs determined by the current stress relaxation method not only makes a direct comparison between model predictions and measured macroscopic properties of BMGs possible, but also offers a clear physical explanation for some unique mechanical responses of metallic glasses. The link between microinstability and macrodeformation further confirms that the macro plasticity originates from activation of localized STZs. Any factor affecting the atomic structures in BMGs, either intrinsic or extrinsic, would alter the distribution of activation energy, thereby resulting in a different macroscopic deformation behavior. Higher cooling rates that are normally applied to fabricate thinner wires were confirmed to increase capability of plastic deformation in amorphous systems, ${ }^{29}$ thus leading to the lower average STZ energy barrier in thinner wires.

As deformation proceeds, STZs with small energy barriers will be activated first and the macroscopic yielding would take place as the portion of the activated STZs reaches a critical value. In the small sized samples, many STZs possess small activation energy barriers and the apparent yielding occurs at the point when the total plastic strain originated form localized shearing of STZs becomes appreciable on the macroscopic stress-strain curve. As the sample is further stretched, STZs with large activation energies start to be activated and irreversible plasticity is created. For large specimens, however, the average activation energy $\bar{V}$ is quite higher and its distribution is also much broader [see Fig. 1(b)]. Thus, it is difficult for the STZs in large amorphous samples to be activated to reach the critical point for apparent yield. Consequently, almost no plastic deformation can be observed for these samples up to stress levels high enough for the initiation of shear bands, which eventually leads to the catastrophic fracture of the specimen. This observation is consistent with recent experimental observations that formation of shear bands becomes easier in larger glassy micropillars under stress. ${ }^{30}$ Therefore, tensile behavior of amorphous solids is closely related to the microplastic instability dictated by the characteristic atomic arrangements in individual STZs. With the availability of the aforementioned material parameters corresponding to localized atomic arrangements, our model can readily be used to predict the macroscopic responses of metallic glasses under more complicated, such as cyclic or multiaxis, loading conditions.

\section{B. Effects of the preset strain on the activation energy}

Different preset strains of $2.2 \%$ and $2.5 \%$ were selected for the stress relaxation experiments of the amorphous wires with a diameter of $125 \mu \mathrm{m}$, which corresponds to a different onset stress. Stress relaxation with a smaller preset stress shows slightly slower stress reduction and a higher equilibrium stress, as shown in Fig. 4(a). The resultant activation energy distributions under the different applied stresses are shown in Fig. 4(b). Activation energy derived from the stress relaxation under a higher applied stress exhibits a lower average value and a slightly narrower distribution, indicating 
that the plastic deformation has created a certain amount of STZs with low energy barriers. As suggested by previous theoretical results, $3,28,31$ a higher external stress could lead the system to a less stable state, which results in the observed small difference in the activation energy. Nevertheless, the fitting results with Eq. (8) using the information derived from these two relaxation tests are insignificant.

Experimental work has confirmed the increase in free volume during deformation which may be the cause of localized softening. ${ }^{23}$ Moreover, many theoretical and simulation work suggested that the applied external stress would reduce the local energy barrier for a shear transition in metallic glasses. ${ }^{3,31}$ However, the descriptions were mostly based on a mean field approximation and the obtained results were the average activation energy. In a more realistic description with distributed plastic events, the dynamic variation in the activation energy spectrum needs more investigation. Based on the aforementioned model, a less tedious and more convenient method can be utilized to estimate the dynamic variation in the plastic events during deformation.

\section{CONCLUSIONS}

In summary, we have proposed a novel approach to build a quantitative link between the microinstability and macroscopic deformation behavior of metallic glasses. Our analysis indicates that microplastic instability of all the individual STZs, with activation energy statistically distributed, governs the macroscopic deformation behavior of amorphous solids. Our model successfully reproduces the macroscopic stressstrain curves of amorphous solids at different length scales and hence offers new insights into understanding the actual deformation mechanism of metallic glasses.

\section{ACKNOWLEDGMENTS}

This research was supported in part by National Natural Science Foundation of China (Grant No. 50725104), the 973 program (Grant No. 2007CB613903), and the program of Introducing Talents of Discipline to Universities (Project No. B07003). Thanks are due to Dr. A. D. Drozdov at Ben-
Gurion University of the Negev, Israel, for fruitful discussion on the modeling work.

${ }^{1}$ F. Spaepen, Acta Metall. 25, 407 (1977).

${ }^{2}$ A. S. Argon, Acta Metall. 27, 47 (1979).

${ }^{3}$ W. L. Johnson and K. Samwer, Phys. Rev. Lett. 95, 195501 (2005).

${ }^{4}$ M. L. Falk and J. S. Langer, Phys. Rev. E 57, 7192 (1998).

${ }^{5}$ M. H. Cohen and D. Turnbull, J. Chem. Phys. 31, 1164 (1959).

${ }^{6}$ M. Zink, K. Samwer, W. L. Johnson, and S. G. Mayr, Phys. Rev. B 73, 172203 (2006).

${ }^{7}$ E. Bouchbinder, J. S. Langer, and I. Procaccia, Phys. Rev. E 75, 036107 (2007).

${ }^{8}$ C. Maloney and A. Lemaître, Phys. Rev. Lett. 93, 195501 (2004).

${ }^{9}$ A. C. Lund and C. A. Schuh, Acta Mater. 51, 5399 (2003).

${ }^{10}$ J. L. Barrat and J. J. de Pablo, MRS Bull. 32, 941 (2007).

${ }^{11}$ Y. H. Liu, G. Wang, R. J. Wang, D. Q. Zhao, M. X. Pan, and W. H. Wang, Science 315, 1385 (2007).

${ }^{12}$ K. B. Kim, J. Das, F. Baier, M. B. Tang, W. H. Wang, and J. Eckert, Appl. Phys. Lett. 88, 051911 (2006).

${ }^{13}$ C. E. Lekka, A. Ibenskas, A. R. Yavari, and G. A. Evangelakis, Appl. Phys. Lett. 91, 214103 (2007).

${ }^{14}$ J. G. Wang, D. Q. Zhao, M. X. Pan, C. H. Shek, and W. H. Wang, Appl. Phys. Lett. 94, 031904 (2009).

${ }^{15}$ Y. Q. Cheng, A. J. Cao, H. W. Sheng, and E. Ma, Acta Mater. 56, 5263 (2008).

${ }^{16}$ P. Schall, D. A. Weitz, and F. Spaepen, Science 318, 1895 (2007).

${ }^{17}$ C. Maloney and A. Lemaître, Phys. Rev. Lett. 93, 016001 (2004).

${ }^{18}$ N. P. Bailey, J. Schiøtz, A. Lemaître, and K. W. Jacobsen, Phys. Rev. Lett. 98, 095501 (2007).

${ }^{19}$ Y. Wu, H. X. Li, G. L. Chen, X. D. Hui, B. Y. Wang, and Z. P. Lu, Scr. Mater. 61, 564 (2009).

${ }^{20}$ B. Derrida, Phys. Rev. Lett. 45, 79 (1980).

${ }^{21}$ S. Matsuoka, Relaxation Phenomena in Polymers, 2nd ed. (Hanser, New York, 1992).

${ }^{22}$ Z. F. Zhang, J. Eckert, and L. Schultz, Acta Mater. 51, 1167 (2003).

${ }^{23}$ K. M. Flores, E. Shere, A. Bharathula, H. Chen, and Y. C. Jean, Acta Mater. 55, 3403 (2007).

${ }^{24}$ L. D. Landau and E. M. Lifshitz, Theory of Elasticity, 3rd ed. (Pergamon, London, 1986).

${ }^{25}$ D. Pan, A. Inoue, T. Sakurai, and M. W. Chen, Proc. Natl. Acad. Sci. U.S.A. 105, 14769 (2008).

${ }^{26}$ J. B. Puthoff, D. S. Stone, and H. B. Cao, and P. M. Voyles, Mater. Res. Soc. Symp. Proc. 1048, Z03 (2008).

${ }^{27}$ S. G. Mayr, Phys. Rev. Lett. 97, 195501 (2006).

${ }^{28}$ D. Rodney and C. A. Schuh, Phys. Rev. Lett. 102, 235503 (2009).

${ }^{29}$ Y. F. Shi and M. L. Falk, Phys. Rev. Lett. 95, 095502 (2005).

${ }^{30}$ C. A. Volkert, A. Donohue, and F. Spaepen, J. Appl. Phys. 103, 083539 (2008).

${ }^{31}$ D. L. Malandro and D. J. Lacks, J. Chem. Phys. 110, 4593 (1999). 